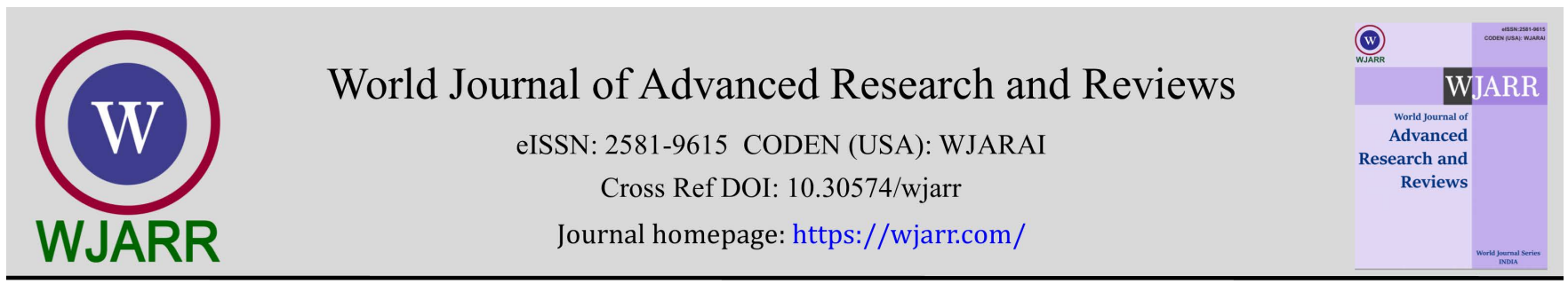

(RESEARCH ARTICLE)

\title{
Dysglycemia in patients with Acromegaly and Cushing's syndrome
}

\author{
Hind Asbar*, Fatima Zahra El Jaafari, Sana Rafi, Ghizlane El Mghari and Nawal El Ansari \\ Department of Endocrinology, Diabetes, Metabolic diseases and Nutrition, Mohammed VI university hospital of Marrakech, \\ Faculty of medicine and pharmacy of Marrakech, Cadi Ayyad University, Marrakech, Morocco.
}

World Journal of Advanced Research and Reviews, 2022, 13(01), 508-512

Publication history: Received on 10 December 2021; revised on 13 January 2022; accepted on 15 January 2022

Article DOI: https://doi.org/10.30574/wjarr.2022.13.1.0054

\begin{abstract}
Introduction: Acromegaly and Cushing's syndrome are endocrine disorders associated to frequent metabolic abnormalities. The aim of our study was to assess the prevalence of prediabetes and overt diabetes in patients presenting with acromegaly and Cushing's syndrome and to find the association between glucose intolerance, age, gender, sex, body mass index, the level of activity of the disease and the duration of symptoms.
\end{abstract}

Patients and methods: This is a retrospective and descriptive review of patient records presenting acromegaly and Cushing's syndrome. Seventy-eight patients were included. Patients were grouped into normoglycemic and dysglycemic based on fasting blood glucose and hemoglobin A1C. Data of age, sex, gender, levels of fasting blood glucose and hemoglobin A1C were reviewed before and after treatment of acromegaly or Cushing's syndrome.

Results: Major risk factors for dysglycemia included: a mean age of 43 years in acromegaly and 28.6 years in Cushing's syndrome patients, a male predominance in acromegalic patients and female predominance in Cushing's syndrome ones, body mass index and duration of disease was higher in dysglycemic Cushing's syndrome patients. Mean IGF-1 levels were higher among the dysglycemic than normoglycemic acromegalic patients. A decrease in mean HbA1c after surgical or medical treatment was also noted in both patients with Cushing's syndrome and acromegaly.

Conclusion: Dysglycemia in the form of prediabetes and diabetes are frequently observed in patients with acromegaly and hypercortisolism. Early diagnosis of glucose metabolism abnormalities in the course of the disease can decrease complications. Treatment modalities have varied impacts on glucose metabolism.

Keywords: Acromegaly; Cushing's syndrome; Prediabetes; Diabetes mellitus; Risk factors

\section{Introduction}

Acromegaly and Cushing's syndrome (CS) are endocrine disorders associated to frequent metabolic abnormalities contributing in premature morbidity and mortality. Among metabolic disorders, dysglycemia in the form of prediabetes and diabetes mellitus are frequently observed in patients with acromegaly and hypercortisolism. A sustained excess of growth hormone $(\mathrm{GH})$ and cortisol, that are antagonistic to insulin, is responsible of insulin resistance and impaired glucose metabolism. The aim of our study was to assess the prevalence of prediabetes and overt diabetes in patients presenting with acromegaly and CS at the endocrinology department of Mohammed VI university hospital of Marrakech. The aim was also to find the association between glucose intolerance, age, sex, body mass index (BMI), the level of activity of the disease and the duration of symptoms.

\footnotetext{
* Corresponding author: Hind Asbar

Department of Endocrinology, Diabetes, Metabolic diseases and Nutrition, Mohammed VI university hospital of Marrakech, Faculty of medicine and pharmacy of Marrakech, Cadi Ayyad University, Marrakech, Morocco.

Copyright (C) 2022 Author(s) retain the copyright of this article. This article is published under the terms of the Creative Commons Attribution Liscense 4.0.
} 


\section{Patients and methods}

We retrospectively reviewed data collected from records of patients that presented with acromegaly and CS at the Endocrinology and Metabolic Diseases department of Mohammed VI University Hospital of Marrakech.

For acromegaly, confirmed cases fulfilling the clinical features and endocrine society diagnostic criteria were included. Patients with elevated or equivocal serum IGF-1 levels matched for age and sex, and confirmation of the diagnosis by finding lack of suppression of $\mathrm{GH}$ to $<0,3 \mu \mathrm{g} / \mathrm{L}$ following documented hyperglycemia during an oral glucose load.

For CS, the diagnosis was based, according to international criteria, on at least two of the following: high daily urinary free cortisol (UFC) levels, absent cortisol suppression after $1 \mathrm{mg}$ overnight dexamethasone suppression test and lack of cortisol rhythm.

With regards to dysglycemia, the patients were subdivided into three groups, according to the diagnostic criteria of the American Diabetes Association (ADA): group 1 with normoglycemia defined as fasting blood glucose (FBG) of less than $1 \mathrm{~g} / \mathrm{l}$, and/or a two-hour post 75-gram glucose load (OGTT) of less than 1,4 g/l and/or HbA1C of less than 5.7\%, group 2 with prediabetes defined as an impaired fasting glycemia between 1 and 1,25 g/l (IFG) and/or impaired glucose tolerance (IGT) with a two-hour post-OGTT-glucose between 1,4 and 1,99 g/l and/or HbA1C between 5.7\% to $6.5 \%$, and finally group 3 with diabetes mellitus which is defined as a FBG greater than 1,26 g/l or a two-hour post-OGTTglucose of $2 \mathrm{~g} / \mathrm{l}$ or more and $\mathrm{HbA} 1 \mathrm{C}$ greater than $6.5 \%$.

Age, sex, body mass index (BMI), fasting glycaemia, HbA1C and duration since diagnosis of acromegaly and CS were evaluated in our patients. The occurrence of dysglycemia before or after having been diagnosed by these diseases was also assessed.

To demonstrate the relationship between the severity of acromegaly and the onset of diabetes, the level of IGF1 was evaluated.

To assess the impact of surgery and/or medical therapy on glucose metabolism in both acromegaly and CS, patients were followed up with $\mathrm{HbA1c}$, and diabetes control was considered improved when the HbA1C level would decrease at least by $1 \%$.

\section{Results}

A total of 78 patients were enrolled in our study: 44 diagnosed with acromegaly and 34 with CS.

Figures 1 and 2 illustrate the percentage of patients with prediabetes and diabetes in these two populations :

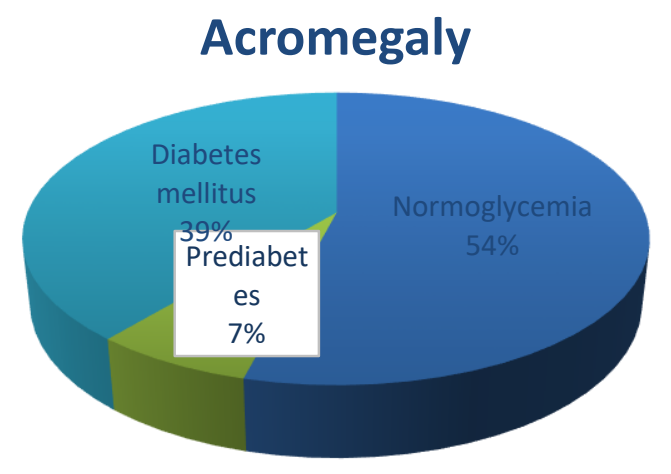

normoglycemia Prediabetes Diabetes mellitus

\section{Cushing's syndrome}

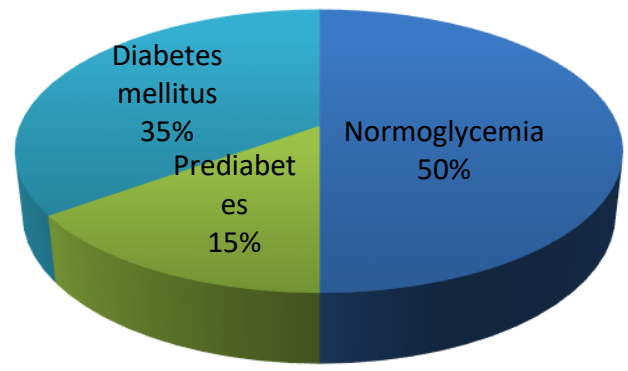

Normoglycemia $\square$ Prediabetes Diabetes mellitus

Figure 1-2 Prevalence of normoglycemia, prediabetes, and diabetes mellitus in patients with acromegaly and Cushing's syndrome 
The clinical, phenotypic, and biochemical features grouped according to normoglycemic and dysglycemic profil of patients with acromegaly and CS are listed out in Table 1:

Table 1 Comparative characteristics of normoglycemic and dysglycemic patients with Acromegaly and Cushing's syndrome. Age, BMI, initial HbA1c, post treatment HbA1c, FBG and estimated duration of the disease are expressed as mean values.

\begin{tabular}{|c|c|c|c|c|}
\hline \multirow[b]{2}{*}{ Parameters } & \multicolumn{2}{|c|}{ Patients with Acromegaly } & \multicolumn{2}{|c|}{ Patients with Cushing's syndrome } \\
\hline & $\begin{array}{l}\text { Normoglycemic } \\
\text { patients }\end{array}$ & $\begin{array}{l}\text { Dysglycemic } \\
\text { patients }\end{array}$ & $\begin{array}{l}\text { Normoglycemic } \\
\text { patients }\end{array}$ & $\begin{array}{c}\text { Dysglycemic } \\
\text { patients }\end{array}$ \\
\hline Patients: n (\%) & $24(55 \%)$ & $20(45 \%)$ & $17(50 \%)$ & $17(50 \%)$ \\
\hline Previously known dysglycemia n (\%) & - & $7(15 \%)$ & - & $5(14 \%)$ \\
\hline Newly diagnosed dysglycemia n (\%) & - & $13(30 \%)$ & - & $12(36 \%)$ \\
\hline Mean age (years) & 38.3 & 43 & 42.1 & 28.6 \\
\hline Male/Female & $10 / 14$ & $12 / 8$ & $4 / 12$ & $7 / 11$ \\
\hline Mean BMI $\left(\mathrm{Kg} / \mathrm{m}^{2}\right)$ & 27.6 & 28.8 & 28.1 & 33.8 \\
\hline Mean Initial HBa1c (\%) & - & 8.9 & - & 7.2 \\
\hline Mean Post treatment HBa1c (\%) & - & 6.2 & - & 6.1 \\
\hline Mean fasting blood glucose (g/l) & 0.9 & 1.6 & 0.8 & 1.7 \\
\hline $\begin{array}{l}\text { Mean Estimated duration of the } \\
\text { disease (months) }\end{array}$ & 15 & 16 & 22 & 48.4 \\
\hline
\end{tabular}

In Acromegaly, 7\% (3/44) of patients had prediabetes and 35\% (15/44) were diabetic. No significant differences were found in what concerns the BMI, and the duration of the disease, except for mean age (43 years for dysglycemic patients and 38.3 years for normoglycemic patients).

Among dysglycemic patients, a male predominance (8M/10F) was found and the percentage of patients with previously known dysglycemia/diabetes was $15 \%$ and for the ones with newly diagnosed dysglycemia it was 35\% of patients. Mean FBG in normoglycemic patients was $0.9 \mathrm{~g} / \mathrm{l}$ and in dysglycemic patients was $1.6 \mathrm{~g} / \mathrm{l}$. Our study showed that among acromegalic patients, the IGF-1 levels were between 100 and $300 \mathrm{ng} / \mathrm{ml}$ in normoglycemic patients and $>400 \mathrm{ng} / \mathrm{ml} \mathrm{in}$ dysglycemic patients. All dyglycemic patients experienced a decrease in mean HbA1c after surgical or medical treatment, with HbA1c decreasing from $8.9 \%$ to $6.2 \%$.

In Cushing's syndrome, 15\% (5/34) of patients had pre-diabetes and 35\% (12/34) had diabetes mellitus, with a female predominance $(7 \mathrm{M} / 11 \mathrm{~F})$ in this population. Several differences were noted in some of the parameters studied in normomoglycemic patients and in those with dysglycemia, notably and respectively, in age (42.1 vs 28.6 years), BMI ( $28.1 \mathrm{vs} 33.8 \mathrm{~kg} / \mathrm{m}^{2}$ ), and duration of disease ( $22 \mathrm{vs} 48.4$ months). For the dysglycemic patients the percentage of patients with previously known diabetes was $14 \%$ and for the ones with newly diagnosed diabetes it was $36 \%$. A decrease in mean HbA1c after surgical and/or medical treatment was also noted in patients with CS, with HbA1c decreasing from 7.2 to $6.1 \%$. Mean FBG glucose in normoglycemic patients was $0.8 \mathrm{~g} / \mathrm{l}$ and in dysglycemic patients at $1.7 \mathrm{~g} / \mathrm{l}$.

\section{Discussion}

Varying degrees of glucose imbalance ranging from moderate degree of impaired fasting glucose and/or impaired glucose tolerance to overt diabetes mellitus are seen secondarily in endocrine diseases. In acromegaly, studies report a prevalence of diabetes mellitus between 12 and $52 \%$ and a prevalence of prediabetes between 16 and $46 \%$ of cases [1]. In Cushing's syndrome (CS), diabetes and prediabetes prevalence was reported to be of 13 to $35 \%$ and 36 to $94 \%$ respectively [2]. This variability in the prevalence of dysglycemia is due to the small number of large studies with acromegaly and CS patients, also by the differently used criteria and guidelines for diagnosing diabetes especially in the less recent studies that were conducted before currently accepted criteria for diabetes diagnosis [2,3]. In our study, the 
American Diabetes Association (ADA) guidelines for diagnosing diabetes and prediabetes were used [4]. Our results revealed a high prevalence of diabetes (39\%) and a prevalence of prediabetes of $7 \%$ in acromegalic patients. In CS patients, the prevalence of diabetes was also increased (35\%) and the prevalence of prediabetes was higher (15\%) than in the acromegalic population.

Abnormal glucose homeostasis in these two endocrinopathies is due to hormone excess. In acromegaly, GH excess stimulates gluconeogenesis and promotes insulin resistance through lipolysis which results in liberation of fatty acids and their competition with the use of glucose in the muscle. Subsequent hyperinsulinemia is a compensating mechanism from the pancreatic $\beta$-cell allowing to preserve normal glucose tolerance in some patients [5]. Concerning CS, cortisol excess exerts anti-insulin effects in the liver, skeletal muscle and adipose tissue leading to insulin resistance. Glucocorticoids induce impairment of the uptake and metabolism of glucose by pancreatic $\beta$-cells with consequent $\beta$ cell dysfunction. The latter mechanism, is an important factor of the development of glucose intolerance in patients with CS since it may prevent the compensatory increase of insulin secretion in response to insulin resistance induced by cortisol excess [6].

The development of dysglycemia in acromegalic and CS patients is multifactorial, related not only to chronic exposure to high hyperglycemic hormone levels but also to age, gender, family history of diabetes, duration of the disease and body mass index (BMI) [7,8]. Studies investigating the impact of these parameters on the incidence of dysglycemia both in acromegaly and CS patients report conflicting results. While some studies showed a significant correlation between advanced age, higher BMI, higher disease duration and female gender and the development of dysglycemia [2,9-11], others found no significant correlation [12,13]. Our study revealed that the age of dysglycemic acromegalic patients was slightly higher than of normoglycemic patients; however, in the CS group dysglycemic patients were unexpectedly younger than normoglycemic patients (mean age of 28 years vs of 42 years in normoglycemic patients). In accordance with previous studies, our results showed that BMI and duration of disease was higher in dysglycemic patients in CS group.

Disease activity and its impact on glucose imbalance in acromegaly and CS is controversial. Some studies revealed that IGF1 levels and tumor size are more likely to be associated with insulin resistance which was the case in our study as IGF1 levels were higher in dysglycemic patients [14,15]. In contrast, the French acromegaly registry did not show any significant association between the level of IGF-1 and GH and the risk of diabetes [7]. In CS patients, a correlation between the degree of hypercortisolism, as assessed by urinary and serum cortisol values, and fasting glucose was demonstrated $[8,16]$.

Based on the above, we suggest that abnormal glucose metabolism is a resultant of conventional risk factors for diabetes aggravated by the sustained hormone excess seen in both acromegalic and CS patients. Disease management has also its effect on glycemic imbalance ; studies showed glycemic control improvement with surgical and medical therapy of acromegaly [17-19]. Our study was in accordance with these data as glycemic control based on HbA1c levels improved post-therapy.

\section{Conclusion}

A high prevalence of dysglycemia in acromegaly and Cushing's syndrome was found in our study. The occurrence of glucose imbalance depends on several factors, hormone excess and disease activity but also on conventional risk factors of diabetes though inconstantly correlated to development of glucose tolerance impairments. Larger studies are needed to assess on a larger scale the impact of various parameters on diabetes and prediabetes occurrence.

\section{Compliance with ethical standards}

\section{Disclosure of conflict of interest}

No conflict of interest.

\section{Statement of informed consent}

Informed consent was obtained from all individual participants included in the study. 


\section{References}

[1] Dreval AV, Trigolosova IV, Misnikova IV, Kovalyova YA, Tishenina RS, Barsukov IA, et al. Prevalence of diabetes mellitus in patients with acromegaly. Endocr Connect. 1 juin 2014; 3(2): 93-8.

[2] Biering H, Knappe G, Gerl H, Lochs H. [Prevalence of diabetes in acromegaly and Cushing syndrome]. Acta Med Austriaca. 2000; 27(1): 27-31.

[3] Stelmachowska-Banaś M, Zdunowski P, Zgliczyński W. Abnormalities in glucose homeostasis in acromegaly. Does the prevalence of glucose intolerance depend on the level of activity of the disease and the duration of the symptoms? Endokrynol Pol. Févr. 2009; 60(1): 20-4.

[4] American Diabetes Association. Classification and Diagnosis of Diabetes: Standards of Medical Care in Diabetes2021. Diabetes Care. janv 2021; 44(1): S15-33.

[5] Hannon AM, Thompson CJ, Sherlock M. Diabetes in Patients with Acromegaly. Curr Diab Rep. févr. 2017; 17(2): 8.

[6] Mazziotti G, Formenti AM, Frara S, Maffezzoni F, Doga M, Giustina A. Diabetes in Cushing Disease. Curr Diab Rep. mai. 2017; 17(5): 32.

[7] Fieffe S, Morange I, Petrossians P, Chanson P, Rohmer V, Cortet C, et al. Diabetes in acromegaly, prevalence, risk factors, and evolution: data from the French Acromegaly Registry. Eur J Endocrinol. Juin. 2011; 164(6): 877-84.

[8] Giordano C, Guarnotta V, Pivonello R, Amato MC, Simeoli C, Ciresi A, et al. Is diabetes in Cushing's syndrome only a consequence of hypercortisolism? Eur J Endocrinol. 1 févr. 2014; 170(2): 311-9.

[9] Nabarro JD. Acromegaly. Clin Endocrinol (Oxf). Avr. 1987; 26(4): 481-512.

[10] Kreze A, Mikulecky M, Moravcik M. Factors influencing the development of glucose intolerance in Cushing syndrome. Acta Med Austriaca. 1995; 22(5): 110-2.

[11] Espinosa-de-los-Monteros AL, González B, Vargas G, Sosa E, Mercado M. Clinical and biochemical characteristics of acromegalic patients with different abnormalities in glucose metabolism. Pituitary. 1 sept 2011; 14(3): 231-5.

[12] Kapoor S. Acromegaly. N Engl J Med. 22 mars 2007; 356(12): 1274-5; author reply 1275-1276.

[13] Katznelson L, Laws ER Jr, Melmed S, Molitch ME, Murad MH, Utz A, et al. Acromegaly: An Endocrine Society Clinical Practice Guideline. J Clin Endocrinol Metab. 1 nov 2014; 99(11): 3933-51.

[14] Niculescu D, Purice M, Coculescu M. Insulin-like growth factor-I correlates more closely than growth hormone with insulin resistance and glucose intolerance in patients with acromegaly. Pituitary. 1 juin 2013; 16(2): 168-74.

[15] Mercado M, Abreu C, Vergara-López A, González-Virla B, Espinosa-de-los-Monteros A-L, Sosa-Eroza E, et al. Surgical and Pharmacological Outcomes in Acromegaly: Real-Life Data From the Mexican Acromegaly Registry. J Clin Endocrinol Metab. 1 déc 2020; 105(12): e4567-76.

[16] Mancini T, Kola B, Mantero F, Boscaro M, Arnaldi G. High cardiovascular risk in patients with Cushing's syndrome according to 1999 WHO/ISH guidelines. Clin Endocrinol (Oxf). déc 2004; 61(6): 768-77.

[17] Khan SA, Ram N, Masood MQ. Patterns of Abnormal Glucose Metabolism in Acromegaly and Impact of Treatment Modalities on Glucose Metabolism. Cureus, 2021; 13(3).

[18] Ronchi CL, Varca V, Beck-Peccoz P, Orsi E, Donadio F, Baccarelli A, et al. Comparison between Six-Year Therapy with Long-Acting Somatostatin Analogs and Successful Surgery in Acromegaly: Effects on Cardiovascular Risk Factors. J Clin Endocrinol Metab. 1 janv 2006; 91(1): 121-8.

[19] Schernthaner-Reiter MH, Siess C, Gessl A, Scheuba C, Wolfsberger S, Riss P, et al. Factors predicting long-term comorbidities in patients with Cushing's syndrome in remission. Endocrine. 2019; 64 (1): 157-68. 